\title{
On coupled systems of fractional impulsive differential equations by using a new Caputo-Fabrizio fractional derivative
}

\author{
Ahmed Boudaoui, Abdeldjalil Slama
}

\begin{abstract}
In this paper, we investigate the existence and uniqueness of solutions for coupled system of Caputo-Fabrizio fractional impulsive differential equations using the fixed point approach in generalized metric spaces. The compactness of solution sets of the system is also investigated. An example is provided to illustrate the developed theory.
\end{abstract}

\section{INTRODUCTION}

The fractional calculus is nowadays a very attractive subject to mathematicians, and many different forms of fractional differential operators were introduced. To increase the applicability of the fractional calculus, some authors proposed a new type of fractional derivatives possessing different kernels. The most used definitions proposed by Riemann-Liouville and the first Caputo version has the weakness that their kernel had singularity [9]. A recent new definition of fractional derivative without singular kernel has been provided by Caputo and Fabrizio [15] and its properties were discussed in [22]. A fractional order derivative is important for developing mathematical models in many scientific and engineering disciplines (see [7]). Several qualitative results for different classes of differential equations with different types of fractional integral and derivatives were obtained in [1, 3, 17, 23, 25, 27, 28].

Thereby, many evolution processes in physics, chemical technology, population dynamics, and natural sciences may change state abruptly or be subject to short-term perturbations. These perturbations may be seen as impulses, which led to define a class of differential equations known as impulsive differential equations. We refer to $[12,21]$ for an introduction to the theory of impulsive differential equations. Recently, several papers have been devoted to study the solutions of differential equations with new types

2010 Mathematics Subject Classification. Primary: 34A08; Secondary: 34A37.

Key words and phrases. Fractional impulsive differential equations, Caputo-Fabrizio Fractional Derivative, Fixed point principle, Generalized metric space.

Full paper. Received 28 October 2019, revised 31 January 2019, accepted 14 April 2020, available online 12 June 2020. 
of fractional derivatives and their applications, see $[8,5,10,14]$ and the references therein.

Coupled systems of fractional-order differential equations appear in the mathematical formulation of several real areas like physics, engineering, chemistry, biology, viscoelasticity etc. [11, 24]. Recently, the study of existence and uniqueness of solutions of coupled systems of fractional order differential equations has also attracted some attention. Alsaedi et al. [5] examined the existence of solutions for a coupled system of time-fractional differential equations and inclusions by using the new Caputo-Fabrizio fractional derivative. Recently, Berrezoug et al. [13] studied the existence and uniqueness, continuous dependence on initial conditions and the boundedness of solutions for a system of impulsive differential equations using the fixed point approach in vector Banach spaces. Very recently, Chalishajar and Kumar [16] investigated the existence and uniqueness of the solutions to a fractional order nonlinear coupled system with integral boundary conditions. Furthermore, Ulam's type stability of the proposed coupled system is studied. For more results on the study of coupled systems of fractional differential equations, we refer to $[2,5,4,20,13]$ and the references therein.

In this paper, we consider the following coupled system of fractional impulsive differential equations involving the Caputo-Fabrizio fractional derivative:

$$
\left\{\begin{array}{l}
\left(D_{t}^{\alpha} x\right)(t)=f_{1}(t, x, y), \\
\left(D_{t}^{\beta} y\right)(t)=f_{2}(t, x, y), \\
\Delta x\left(t_{k}\right)=x\left(t_{k}^{+}\right)-x\left(t_{k}^{-}\right)=I_{k}\left(x\left(t_{k}\right), y\left(t_{k}\right)\right), \quad k=1, \ldots, m, \\
\Delta y\left(t_{k}\right)=y\left(t_{k}^{+}\right)-y\left(t_{k}^{-}\right)=\bar{I}_{k}\left(x\left(t_{k}\right), y\left(t_{k}\right)\right), \\
x(0)=x_{0}, \quad y(0)=y_{0},
\end{array}\right.
$$

where $D_{t}^{\alpha}, D_{t}^{\beta}$ are the Caputo-Fabrizio fractional derivative of order $\alpha$ and $\beta, 0<\alpha, \beta<1$. Here $0=t_{0} \leq t_{1} \leq \cdots \leq t_{m} \leq t_{m+1}=T$, $\Delta x\left(t_{k}\right)=I_{k}\left(x\left(t_{k}^{-}\right)\right)=x\left(t_{k}^{+}\right)-x\left(t_{k}^{-}\right), x\left(t_{k}^{+}\right)=\lim _{h \rightarrow 0} x\left(t_{k}+h\right)$ and $x\left(t_{k}^{-}\right)=$ $\lim _{h \rightarrow 0} x\left(t_{k}-h\right)$ represent the right and left limits of $x(t)$ at $t=t_{k}$ respectively. $x_{0}, y_{0} \in \mathbb{R}, f_{1}, f_{2}: J \times \mathbb{R} \times \mathbb{R} \rightarrow \mathbb{R}$ are continuous functions and $I_{k}$, $\bar{I}_{k} \in C(\mathbb{R} \times \mathbb{R}, \mathbb{R})$ are given functions.

The rest of this paper is organized as follows. In Section 2, we introduce all the background material used in this paper such as definition of CaputoFabrizio derivatives of fractional order and some properties of generalized Banach spaces and fixed point theory. In Sections 3 and 4, using Perov's and Schaefer fixed point type theorems in generalized Banach spaces, we prove some existence and compactness results for problem (1). An example 
is given to demonstrate the application of our main results in section 4 . Finally, a conclusion is given in section 5 .

\section{Preliminaries}

For completeness, in this section, we will give some notations, basic definitions and some fundamental facts of Caputo-Fabrizio derivatives of fractional order which can be found in [15] and [22].

Definition 1. The $\alpha$ order Caputo-Fabrizio time fractional differential derivative of the function $u$ is written as

$$
\left({ }^{C F} D_{t}^{\alpha} u\right)(t)=\frac{(2-\alpha) M(\alpha)}{2(1-\alpha)} \int_{0}^{t} \exp \left[-\frac{\alpha(t-s)}{1-\alpha}\right] u^{\prime}(s) d s, \quad t \geq 0,
$$

where $M(\alpha)$ represents a normalization function such that $M(0)=M(1)=$ $1,0<\alpha<1$, and $u \in H^{1}(0, b), b>0$.

Note that, according to the definition 1, the new definition of fractional derivative is zero when $u$ is constant, as in the usual Caputo fractional time derivative, but contrary to the usual Caputo fractional time derivative, the kernel does not have singularity for $t=s[15,22]$.

The notion of Caputo-Fabrizio time-fractional integral is,

Definition 2. Let $0<\alpha<1$. the fractional integral of order $\alpha$ of a function $f$ is defined by,

$$
\left({ }^{C F} I_{t}^{\alpha} f\right)(t)=\frac{2(1-\alpha)}{(2-\alpha) M(\alpha)} f(t)+\frac{2 \alpha}{(2-\alpha) M(\alpha)} \int_{0}^{t} f(s) d s, t \geq 0,
$$

where $M(\alpha)$ represents a normalization function and $0<\alpha<1$.

Losada and Nieto [22] note that the fractional integral of Caputo-Fabrizio type of a function of order $0<\alpha<1$ is an average between function $\mathrm{f}$ and its integral of order one.

With imposing

$$
\frac{2(1-\alpha)}{(2-\alpha) M(\alpha)}+\frac{2 \alpha}{(2-\alpha) M(\alpha)}=1
$$

we obtain an explicit formula for $M(\alpha)$,

$$
M(\alpha)=\frac{2}{2-\alpha}, \quad 0<\alpha<1 .
$$

By substituting $M(\alpha)$ in (1), we obtain the definition of the fractional Caputo-Fabrizio derivative of order $0<\alpha<1$ for a function $u$ as follows:

Definition 3. Let $0<\alpha<1$. The fractional Caputo-Fabrizio derivative of order $\alpha$ of a function $u$ is given by

$$
\left({ }^{C F} D_{t}^{\alpha} u\right)(t)=\frac{1}{1-\alpha} \int_{0}^{t} \exp \left(-\frac{\alpha}{1-\alpha}(t-s)\right) u^{\prime}(s) d s, \quad t \geq 0 .
$$


Lemma 1 ([22]). Let $0<\alpha<1$ and $u$ be a solution of the following fractional differential equation,

$$
\left({ }^{C F} D_{t}^{\alpha} u\right)(t)=0,
$$

Then, $u$ is a constant function. The converse, is also true.

Lemma 2 ([22]). Suppose that $f \in L^{1}([0, T])$ and $0<\alpha<1$. Then, the unique solution of the following initial value problem

$$
\begin{gathered}
\left({ }^{C F} D_{t}^{\alpha} u\right)(t)=f(t), \\
u(0)=u_{0} \in \mathbb{R}
\end{gathered}
$$

is given by

$$
u(t)=u_{0}+A_{\alpha}(f(t)-f(0))+B_{\alpha} \int_{0}^{t} f(s) d s,
$$

where $A_{\alpha}=\frac{2(1-\alpha)}{(2-\alpha) M(\alpha)}$ and $B_{\alpha}=\frac{2 \alpha}{(2-\alpha) M(\alpha)}$.

Lemma 3. Let $f:[0, T] \times \mathbb{R} \times \mathbb{R} \rightarrow \mathbb{R}$ be a continuous function with $f(0, x)=0, \forall x \in \mathbb{R}$, and $0<\alpha<1$. Then, a function $x$ is a solution of the following initial value problem

$$
\left\{\begin{aligned}
\left(D_{t}^{\alpha} x\right)(t) & =f(t, x), t \in[0, T], & & t \neq t_{k}, \\
\Delta x(t) & =I_{k}\left(x\left(t_{k}\right)\right), & & k=1, \ldots, m, \\
x(0) & =x_{0}, & &
\end{aligned}\right.
$$

if and only if

$$
x(t)=\left\{\begin{array}{cc}
x_{0}+A_{\alpha} f(t, x)+B_{\alpha} \int_{0}^{t} f(s, x(s)) d s, & t \in\left[0, t_{1}\right], \\
x_{0}+A_{\alpha} f(t, x)+B_{\alpha} \int_{0}^{t} f(s, x(s)) d s & +I_{1}\left(x\left(t_{1}\right)\right), \\
& t \in\left(t_{1}, t_{2}\right], \\
\vdots & \vdots \\
x_{0}+A_{\alpha} f(t, x)+B_{\alpha} \int_{0}^{t} f(s, x(s)) d s & +\sum_{\substack{k\\
}}^{k} I_{i}\left(x\left(t_{i}\right)\right), \\
& t \in\left(t_{k}, t_{k+1}\right] .
\end{array}\right.
$$

Proof. Assume $x$ satisfies (8). If $t \in\left[0, t_{1}\right]$, then

$$
\left(D_{t}^{\alpha} x\right)(t)=f(t, x) \text {. }
$$

Lemma (2) implies

$$
x(t)=x_{0}+A_{\alpha} f(t, x)+B_{\alpha} \int_{0}^{t} f(s, x(s)) d s .
$$

If $t \in\left(t_{1}, t_{2}\right]$ then Lemma (2) implies

$$
x(t)=x\left(t_{1}^{+}\right)+A_{\alpha}\left(f(t, x)-f\left(t_{1}^{+}, x\left(t_{1}^{+}\right)\right)\right)+B_{\alpha} \int_{t_{1}}^{t} f(s, x(s)) d s
$$




$$
\begin{aligned}
= & I_{1}\left(x\left(t_{1}\right)\right)+x\left(t_{1}\right)+A_{\alpha}\left(f(t, x)-f\left(t_{1}^{+}, x\left(t_{1}^{+}\right)\right)\right) \\
& +B_{\alpha} \int_{t_{1}}^{t} f(s, x(s)) d s \\
= & I_{1}\left(x\left(t_{1}\right)\right)+x_{0}+A_{\alpha}\left(f\left(t_{1}, x\left(t_{1}\right)\right)-f\left(0, x_{0}\right)\right) \\
& +B_{\alpha} \int_{0}^{t_{1}} f(s, x(s)) d s \\
& +A_{\alpha}\left(f(t, x)-f\left(t_{1}^{+}, x\left(t_{1}^{+}\right)\right)\right)+B_{\alpha} \int_{t_{1}}^{t} f(s, x(s)) d s \\
= & \left.I_{1}\left(x\left(t_{1}\right)\right)+x_{0}+A_{\alpha}\left(f(t, x)-f\left(0, x_{0}\right)\right)\right) \\
& +B_{\alpha} \int_{0}^{t} f(s, x(s)) d s \\
= & I_{1}\left(x\left(t_{1}\right)\right)+x_{0}+A_{\alpha} f(t, x)+B_{\alpha} \int_{0}^{t} f(s, x(s)) d s .
\end{aligned}
$$

If $t \in\left(t_{2}, t_{3}\right]$, then from Lemma (2) we get

$$
\begin{aligned}
x(t)= & x\left(t_{2}^{+}\right)+A_{\alpha}\left(f(t, x)-f\left(t_{2}^{+}, x\left(t_{2}^{+}\right)\right)\right)+B_{\alpha} \int_{t_{2}}^{t} f(s, x(s)) d s \\
= & I_{2}\left(x\left(t_{2}\right)\right)+x\left(t_{2}\right)+A_{\alpha}\left(f(t, x)-f\left(t_{2}^{+}, x\left(t_{2}^{+}\right)\right)\right) \\
& +B_{\alpha} \int_{t_{2}}^{t} f(s, x(s)) d s \\
= & I_{2}\left(x\left(t_{2}\right)\right)+I_{1}\left(x\left(t_{1}\right)\right)+x_{0}+A_{\alpha}\left(f\left(t_{2}, x\left(t_{2}\right)\right)-f\left(0, x_{0}\right)\right) \\
& +B_{\alpha} \int_{0}^{t_{2}} f(s, x(s)) d s \\
& +A_{\alpha}\left(f(t, x)-f\left(t_{2}^{+}, x\left(t_{2}^{+}\right)\right)\right)+B_{\alpha} \int_{t_{2}}^{t} f(s, x(s)) d s \\
= & \left.I_{1}\left(x\left(t_{1}\right)\right)+I_{2}\left(x\left(t_{2}\right)\right)+x_{0}+A_{\alpha}\left(f(t, x)-f\left(0, x_{0}\right)\right)\right) \\
& +B_{\alpha} \int_{0}^{t} f(s, x(s)) d s \\
= & I_{1}\left(x\left(t_{1}\right)\right)+I_{2}\left(x\left(t_{2}\right)\right)+x_{0}+A_{\alpha} f(t, x)+B_{\alpha} \int_{0}^{t} f(s, x(s)) d s .
\end{aligned}
$$

If $t \in\left(t_{k}, t_{k+1}\right]$, then again from Lemma (2) we get

$$
x(t)=x_{0}+\sum_{i=1}^{k} I_{i}\left(x\left(t_{i}\right)\right)+A_{\alpha} f(t, x)+B_{\alpha} \int_{0}^{t} f(s, x(s)) d s .
$$

Conversely, assume that $x$ satisfies the impulsive fractional integral equation (9). If $t \in\left[0, t_{1}\right]$ then $x(0)=x_{0}$ and we can easily show that

$$
\left.\Delta x\right|_{t=t_{k}}=I_{k}\left(x\left(t_{k}\right)\right), \quad k=1 \ldots, m,
$$


and using the fact that $D^{\alpha} C=0$, where $C$ is a constant and the definition 1 we get

$$
\left(D_{t}^{\alpha} x\right)(t)=f(t, x), t \in\left[0, t_{1}\right] \cup\left(t_{k}, t_{k+1}\right], \quad k=1, \ldots, m .
$$

In the following, we introduce some notations and definitions of generalized metric space.

If $x, y \in \mathbb{R}^{m}$, with $x=\left(x_{1}, \cdots, x_{m}\right)$ and $y=\left(y_{1}, \ldots, y_{m}\right)$, then by $x \leq y$ we mean $x_{i} \leq y_{i}$ for all $i=1, \ldots, m$. Also we set $|x|=\left(\left|x_{1}\right|, \ldots,\left|x_{m}\right|\right)$, $\max (x, y)=\left(\max \left(x_{1}, y_{1}\right), \ldots, \max \left(x_{m}, y_{m}\right)\right)$ and $\mathbb{R}_{+}^{m}=\left\{x \in \mathbb{R}^{m}: x_{i}>0\right\}$. If $c \in \mathbb{R}$, then $x \leq c$ means $x_{i} \leq c$ for each $i=1, \ldots, m$.

Definition 4. Let $X$ be a nonempty set and consider space $\mathbb{R}_{+}^{m}$ endowed with the usual component-wise partial order. The mapping $d: X \times X \rightarrow \mathbb{R}_{+}^{m}$ which satisfies all the usual axioms of the metric is called a generalized metric in Perov's sense and $(X, d)$ is called a generalized metric space.

Let $(X, d)$ be a generalized metric space in Perov's sense with

$$
d(x, y):=\left(\begin{array}{c}
d_{1}(x, y) \\
\vdots \\
d_{m}(x, y)
\end{array}\right), \quad(x, y) \in X \times X .
$$

For $r=\left(r_{1}, \ldots, r_{m}\right) \in \mathbb{R}_{+}^{m}$, we will denote by

$$
\begin{aligned}
B\left(x_{0}, r\right) & =\left\{x \in X: d\left(x_{0}, x\right)<r\right\}= \\
& =\left\{x \in X: d_{i}\left(x_{0}, x\right)<r_{i}, \quad i=1, \ldots, m\right\},
\end{aligned}
$$

the open ball centered in $x_{0}$ with radius $r$ and

$$
\begin{aligned}
B\left(x_{0}, r\right) & =\left\{x \in X: d\left(x_{0}, x\right) \leq r\right\}= \\
& =\left\{x \in X: d_{i}\left(x_{0}, x\right) \leq r_{i}, \quad i=1, \ldots, m\right\},
\end{aligned}
$$

the closed ball centered in $x_{0}$ with radius $r$.

We mention that for a generalized metric space, the notions of open subset, closed set, convergence, Cauchy sequence, and completeness are similar to those in the usual metric spaces.

Definition 5. A square matrix $A$ of real numbers is said to be convergent to zero if and only if $A^{n} \rightarrow 0$ as $n \rightarrow \infty$.

Lemma 4 (see [18]). Let $A \in \mathcal{M}_{m, m}\left(\mathbb{R}_{+}\right)$. Then the following statements are equivalent:

- A is a matrix convergent to zero;

- The eigenvalues of $A$ are in the open unit disc, i.e., $|\lambda|<1$, for every $\lambda \in \mathbb{C}$ with $\operatorname{det}(A-\lambda I)=0$; where $I$ denote the unit matrix of $\mathcal{M}_{m, m}\left(\mathbb{R}_{+}\right)$,

- The matrix $I-A$ is non-singular and $(I-A)^{-1}=I+A+\cdots+A^{n}+\cdots$; 
- The matrix $I-A$ is non-singular and $(I-A)^{-1}$ has nonnegative elements;

- $A^{n} q \rightarrow 0$ and $q A^{n} \rightarrow 0$ as $n \rightarrow \infty$, for any $q \in \mathbb{R}^{m}$.

Some examples of matrices convergent to zero can be found in [18].

Definition 6. Let $(X, d)$ be a generalized metric space. An operator $N$ : $X \rightarrow X$ is said to be contractive if there exists a convergent to zero matrix $A$ such that

$$
d(N(x), N(y)) \leq A d(x, y), \quad \forall x, y \in X .
$$

Theorem 1 (Perov's fixed point theorem, see [26]). . Let $(X, d)$ be a complete generalized metric space and $N: X \rightarrow X$ be a contractive operator with Lipschitz matrix $A$. Then $N$ has a unique fixed point $x^{*}$ and for each $x_{0} \in X$ we have

$$
d\left(N^{k}\left(x_{0}\right), x^{*}\right) \leq A^{k}(I-A)^{-1} d\left(x_{0}, N\left(x_{0}\right)\right), \quad \forall k \in N .
$$

Now, we state Schaefer fixed point theorem type in generalized Banach space.

Theorem 2 ([19]). . Let $X$ be a generalized Banach space and let $G: X \rightarrow$ $X$ be completely continuous. Then, either

(1) the operator equation $x=T x$ has a solution, or

(2) the set

$$
\mathcal{E}=\{x \in X: x=\lambda N(x), \lambda \in(0,1)\}
$$

is unbounded.

\section{Existence And uniqueness of the SOlution}

For a given $T>0$, let $J_{k}=\left(t_{k}, t_{k+1}\right], k=1,2, \ldots, m$. In order to define a solution for problem (1), consider the following space of picewise continuous functions:

$$
\begin{aligned}
P C(J, \mathbb{R})=\{ & y:[0, T] \rightarrow \mathbb{R}, y_{k} \in C\left(J_{k}, \mathbb{R}\right) \text { for } k=0, \ldots m, \\
& \text { and there exist } y\left(t_{k}^{-}\right) \operatorname{and} y\left(t_{k}^{+}\right) \\
& \text {with } \left.y\left(t_{k}\right)=y\left(t_{k}^{-}\right), k=1, \ldots, m\right\}
\end{aligned}
$$

endowed with the norm

$$
\|y\|=\sup _{t \in[0, T]}(|y(t)|) .
$$

It is not difficult to check that $P C(J, \mathbb{R})$ is a Banach space with norm $\|$.$\| .$ Now, we first define the solution to our problem. 
Definition 7. A function $(x, y) \in P C(J, \mathbb{R}) \times P C(J, \mathbb{R})$ is said to be a solution of (1) if and only if

$$
\left\{\begin{aligned}
x(t)= & x_{0}+A_{\alpha} f_{1}(t, x, y)+B_{\alpha} \int_{0}^{t} f_{1}(s, x(s), y(s)) d s \\
& +\sum_{0 \leq t_{k} \leq t} I_{k}\left(x\left(t_{k}\right), y\left(t_{k}\right)\right), \\
y(t)= & y_{0}+A_{\beta} f_{2}(t, x, y)+B_{\beta} \int_{0}^{t} f_{2}(s, x(s), y(s)) d s \\
& +\sum_{0 \leq t_{k} \leq t} \bar{I}_{k}\left(x\left(t_{k}\right), y\left(t_{k}\right)\right) .
\end{aligned}\right.
$$

First, we will list the following hypotheses which will be imposed in our main theorem.

$\left(H_{1}\right)$ There exist constants $l_{i}>0, i=1, \ldots, 4$, such that

$$
\left\|f_{1}(t, x, y)-f_{1}(t, \bar{x}, \bar{y})\right\| \leq l_{1}\|x-\bar{x}\|+l_{2}\|y-\bar{y}\|,
$$

and

$$
\left\|f_{2}(t, x, y)-f_{2}(t, \bar{x}, \bar{y})\right\| \leq l_{3}\|x-\bar{x}\|+l_{4}\|y-\bar{y}\|,
$$

for all $x, \bar{x}, y, \bar{y} \in \mathbb{R}$;

$\left(H_{2}\right)$ There exist constants $a_{1 k}, a_{2 k}, b_{1 k}, b_{2 k} \geq 0, k=1, \ldots, m$, such that

$$
\left\|I_{k}(x, y)-I_{k}(\bar{x}, \bar{y})\right\| \leq a_{1 k}\|x-\bar{x}\|+a_{2 k}\|y-\bar{y}\|,
$$

and

$$
\left\|\bar{I}_{k}(x, y)-\bar{I}_{k}(\bar{x}, \bar{y})\right\| \leq b_{1 k}\|x-\bar{x}\|+b_{2 k}\|y-\bar{y}\|,
$$

for all $x, \bar{x}, y, \bar{y} \in \mathbb{R}$.

We will use the Perov fixed point theorem to prove the existence of a solution to the problem (1).

Theorem 3. Assume that $\left(H_{1}\right)-\left(H_{2}\right)$ are satisfied and the matrix

$$
M=\left(\begin{array}{cc}
A_{\alpha} l_{1}+B_{\alpha} T l_{1}+\sum_{k=1}^{m} a_{1 k} & A_{\alpha} l_{2}+B_{\alpha} T l_{2}+\sum_{k=1}^{m} a_{2 k} \\
A_{\beta} l_{3}+B_{\beta} T l_{3}+\sum_{k=1}^{m} b_{1 k} & A_{\beta} l_{4}+B_{\beta} T l_{4}+\sum_{k=1}^{m} b_{2 k}
\end{array}\right)
$$

converges to zero and $f_{1}(\cdot, 0,0)=f_{2}(\cdot, 0,0)=I_{k}(0,0)=\bar{I}_{k}(0,0)=0$. Then the problem (1) has unique solution.

Proof. Consider the operator $N: P C \times P C \rightarrow P C \times P C$ defined by

$$
N(x, y)=\left(N_{1}(x, y), N_{2}(x, y)\right)
$$

where 


$$
\begin{aligned}
N_{1}(x, y)(t)= & x_{0}+A_{\alpha} f_{1}(t, x, y)+B_{\alpha} \int_{0}^{t} f_{1}(s, x(s), y(s)) d s \\
& +\sum_{0 \leq t_{k} \leq t} I_{k}\left(x\left(t_{k}\right), y\left(t_{k}\right)\right)
\end{aligned}
$$

and

$$
\begin{aligned}
N_{2}(x, y)(t)= & y_{0}+A_{\beta} f_{2}(t, x, y)+B_{\beta} \int_{0}^{t} f_{2}(s, x(s), y(s)) d s \\
& +\sum_{0 \leq t_{k} \leq t} \bar{I}_{k}\left(x\left(t_{k}\right), y\left(t_{k}\right)\right) .
\end{aligned}
$$

We show that $N$ was well defined. Given $(x, y) \in P C \times P C, t \in[0, T]$, we have

$$
\begin{aligned}
\left\|N_{1}(x, y)\right\| \leq & \left\|x_{0}\right\|+A_{\alpha}\left\|f_{1}(t, x, y)\right\|+B_{\alpha} \int_{0}^{t}\left\|f_{1}(s, x(s), y(s))\right\| d s \\
& +\sum_{0 \leq t_{k} \leq t}\left\|I_{k}\left(x\left(t_{k}\right), y\left(t_{k}\right)\right)\right\| \\
\leq & \left\|x_{0}\right\|+A_{\alpha}\left(l_{1}\|x\|+l_{2}\|y\|\right)+B_{\alpha} T\left[l_{1}\|x\|+l_{2}\|y\|\right] \\
& +\sum_{k=1}^{m}\left[\left\|a_{1 k}\right\| x\left\|+a_{2 k}\right\| y \|\right] .
\end{aligned}
$$

Similarly we have

$$
\begin{aligned}
\left\|N_{2}(x, y)\right\| \leq & \left\|y_{0}\right\|+A_{\beta}\left(l_{3}\|x\|+l_{4}\|y\|\right)+B_{\beta} T\left[l_{3}\|x\|+l_{4}\|y\|\right] \\
& +\sum_{k=1}^{m}\left[\left\|b_{1 k}\right\| x\left\|+b_{2 k}\right\| y \|\right]
\end{aligned}
$$

Thus

$$
\begin{aligned}
& \left(\begin{array}{l}
\left\|N_{1}(x, y)\right\| \\
\left\|N_{2}(x, y)\right\|
\end{array}\right) \leq\left(\begin{array}{c}
\left\|x_{0}\right\| \\
\left\|y_{0}\right\|
\end{array}\right)+ \\
& +\left(\begin{array}{cc}
A_{\alpha} l_{1}+B_{\alpha} T l_{1}+\sum_{k=1}^{m} a_{1 k} & A_{\alpha} l_{2}+B_{\alpha} T l_{2}+\sum_{k=1}^{m} a_{2 k} \\
A_{\beta} l_{3}+B_{\beta} T l_{3}+\sum_{k=1}^{m} b_{1 k} & A_{\beta} l_{4}+B_{\beta} T l_{4}+\sum_{k=1}^{m} b_{2 k}
\end{array}\right) \cdot\left(\begin{array}{c}
\|x\| \\
\|y\|
\end{array}\right) .
\end{aligned}
$$

This implies that $N$ is well defined.

Clearly, fixed points of $N$ are solutions of problem (1). We show that $N$ is a contraction. Let $(x, y),(x, y) \in P C \times P C$. Then $\left(H_{1}\right)$ and $\left(H_{2}\right)$ imply

$$
\begin{aligned}
\left\|N_{1}(x, y)-N_{1}(\bar{x}, \bar{y})\right\| \leq & A_{\alpha}\left\|f_{1}(t, x, y)-f_{1}(t, \bar{x}, \bar{y})\right\| \\
& +B_{\alpha} \int_{0}^{t}\left\|f_{1}(s, x, y)-f_{1}(t, \bar{x}, \bar{y})\right\| d s
\end{aligned}
$$




$$
\begin{aligned}
& +\sum_{0 \leq t_{k} \leq t}\left(\left\|I_{k}(x, y)-I_{k}(\bar{x}, \bar{y})\right\|\right. \\
\leq & A_{\alpha}\left[l_{1}\|x-\bar{x}\|+l_{2}\|y-\bar{y}\|\right] \\
& +B_{\alpha} T\left[l_{1}\|x-\bar{x}\|+l_{2}\|y-\bar{y}\|\right] \\
& +\sum_{k=1}^{m}\left[a_{1 k}\|x-\bar{x}\|+a_{2 k}\|y-\bar{y}\|\right] \\
\leq & \left(A_{\alpha} l_{1}+B_{\alpha} T l_{1}+\sum_{k=1}^{m} a_{1 k}\right)\|x-\bar{x}\| \\
& +\left(A_{\alpha} l_{2}+B_{\alpha} T l_{2}+\sum_{k=1}^{m} a_{2 k}\right)\|y-\bar{y}\| .
\end{aligned}
$$

Similarly, we have

$$
\begin{aligned}
\left\|N_{2}(x, y)-N_{2}(\bar{x}, \bar{y})\right\| \leq & \left(A_{\beta} l_{3}+B_{\beta} T l_{3}+\sum_{k=1}^{m} b_{1 k}\right)\|x-\bar{x}\| \\
& +\left(A_{\beta} l_{4}+B_{\beta} T l_{4}+\sum_{k=1}^{m} b_{2 k}\right)\|y-\bar{y}\| .
\end{aligned}
$$

It follows that

$$
\|N(x, y)-N(\bar{x}, \bar{y})\| \leq M\left(\begin{array}{l}
\|x-\bar{x}\| \\
\|y-\bar{y}\|
\end{array}\right), \quad \text { for all }(x, y),(\bar{x}, \bar{y}) \in P C \times P C .
$$

Hence, by Theorem 1 , the operator $N$ has a unique fixed point which is a solution of problem (1).

\section{EXISTENCE AND COMPACTNESS OF SOLUtion SETS}

In this section we prove some existence and compactness results for problem (1) by application of Schaefer fixed point type theorem in generalized Banach spaces. We consider the following hypotheses

$\left(H_{3}\right)$ There exist $c_{1}, c_{2} \geq 0$ such that

$$
\left\|f_{1}(t, x, y)\right\| \leq c_{1}\|x\|+c_{2}\|y\|, \quad \text { for all } \quad x, y, \in \mathbb{R} .
$$

$\left(H_{4}\right)$ There exist $c_{3}, c_{4} \geq 0$ such that

$$
\left\|f_{2}(t, x, y)\right\| \leq c_{3}\|x\|+c_{4}\|y\|, \quad \text { for all } \quad x, y, \in \mathbb{R} .
$$

$\left(H_{5}\right)$ There exist constants $d_{1 k}, d_{2 k}, \geq 0, k=1, \ldots, m$, such that

$$
\left\|I_{k}(x, y)\right\| \leq d_{1 k}\|x\|+d_{2 k}\|y\|, \quad \text { for all } x, y, \in \mathbb{R} .
$$

$\left(H_{6}\right)$ There exist constants $e_{1 k}, e_{2 k} \geq 0, k=1, \ldots, m$, such that

$$
\left\|\bar{I}_{k}(x, y)\right\| \leq e_{1 k}\|x\|+e_{2 k}\|y\|, \quad \text { for all } x, y, \in \mathbb{R} .
$$


Now, we give prove of the existence result of problem (1) by using nonlinear alternative of Schaefer fixed point theorem type in generalized Banach space.

Theorem 4. Assume that the hypotheses $\left(H_{3}\right)-\left(H_{6}\right)$ hold. Then, the problem (1) has a solution defined on $[0, T]$. Moreover, the solution set

$$
S=\{(x, y) \in P C \times P C,(x, y) \text { is solution of }(1)\},
$$

is compact.

Proof. Let $N: P C(J, \mathbb{R}) \times P C(J, \mathbb{R}) \rightarrow P C(J, \mathbb{R}) \times P C(J, \mathbb{R})$ be an operator defined in the proof of Theorem 3.

In order to apply theorem 2 , we first show that $N$ is completely continuous. The proof will be given in several steps.

Step 1. $N(\cdot, \cdot)$ is continuous. Let $\left(x_{n}, y_{n}\right)$ be a sequence such that $\left(x_{n}, y_{n}\right) \rightarrow$ $(x, y) \in P C(J, \mathbb{R}) \times P C(J, \mathbb{R})$ as $n \rightarrow \infty$. Then

$$
\begin{aligned}
\left\|N_{1}\left(x_{n}, y_{n}\right)-N_{1}(x, y)\right\| \leq & A_{\alpha}\left\|f_{1}\left(t, x_{n}, y_{n}\right)-f_{1}(t, x, y)\right\| \\
& +B_{\alpha} \int_{0}^{t}\left\|f_{1}\left(s, x_{n}, y_{n}\right)-f_{1}(t, x, y)\right\| d s \\
& +\sum_{0 \leq t_{k} \leq t}\left(\left\|I_{k}\left(x_{n}, y_{n}\right)-I_{k}(x, y)\right\| .\right.
\end{aligned}
$$

Since $f_{1}$ and $I_{k}$ are a continuous functions. Thus

$$
\left\|N_{1}\left(x_{n}, y_{n}\right)-N_{1}(x, y)\right\| \rightarrow 0 \quad \text { as } n \rightarrow \infty .
$$

Similarly, we can get,

$$
\left\|N_{2}\left(x_{n}, y_{n}\right)-N_{2}(x, y)\right\| \rightarrow 0 \quad \text { as } \quad n \rightarrow \infty .
$$

Thus $N$ is continuous.

Step 2. $N$ maps bounded sets into bounded sets in $P C(J, \mathbb{R}) \times P C(J, \mathbb{R})$. Indeed, it is enough to show that for any $q>0$ there exists a positive constant $l$ such that for each $(x, y) \in B_{q}=\{(x, y) \in P C(J, \mathbb{R}) \times P C(J, \mathbb{R})$ : $\|x\| \leq q,\|y\| \leq q\}$, we have

$$
N(x, y) \leq l=\left(l_{1}, l_{2}\right)
$$

Then for each $t \in[0, T]$, we get

$$
\begin{aligned}
\left\|N_{1}(x, y)\right\| \leq & \left\|x_{0}\right\|+A_{\alpha}\left\|f_{1}(t, x, y)\right\|+B_{\alpha} \int_{0}^{t}\left\|f_{1}(s, x(s), y(s))\right\| d s \\
& +\sum_{0 \leq t_{k} \leq t}\left\|I_{k}\left(x\left(t_{k}\right), y\left(t_{k}\right)\right)\right\| \\
\leq & \left\|x_{0}\right\|+A_{\alpha}\left[c_{1}\|x\|+c_{2}\|y\|\right]+B_{\alpha} T\left[c_{1}\|x\|+c_{2}\|y\|\right] \\
& +\sum_{k=1}^{m}\left[d_{1 k}\|x\|+d_{2 k}\|y\|\right]
\end{aligned}
$$




$$
\begin{aligned}
& \leq\left\|x_{0}\right\|+q\left(A_{\alpha}\left[c_{1}+c_{2}\right]+B_{\alpha} T\left[c_{1}+c_{2}\right]+\sum_{k=1}^{m}\left[d_{1 k}+d_{2 k}\right]\right) \\
& :=l_{1} .
\end{aligned}
$$

Similarly, we have

$$
\begin{aligned}
\left\|N_{2}(x, y)\right\| & \leq\left\|y_{0}\right\|+q\left(A_{\beta}\left[c_{3}+c_{4}\right]+B_{\beta} T\left[c_{3}+c_{4}\right]+\sum_{k=1}^{m}\left[e_{1 k}+e_{2 k}\right]\right) \\
& :=l_{2} .
\end{aligned}
$$

Step 3. $N$ maps bounded sets into equicontinuous sets of $P C([0, T], \mathbb{R}) \times$ $P C([0, T], \mathbb{R})$. Let $B_{q}$ be a bounded set in $P C(J, \mathbb{R}) \times P C(J, \mathbb{R})$ as in Step 2. Let $r_{1}, r_{2} \in J, r_{1}<r_{2}$ and $u \in B_{q}$. Thus we have

$$
\begin{aligned}
& \left\|N_{1}\left(x\left(r_{2}\right), y\left(r_{2}\right)\right)-N_{1}\left(x\left(r_{1}\right), y\left(r_{1}\right)\right)\right\| \leq \\
& \leq A_{\alpha}\left\|f_{1}\left(r_{2}, x\left(r_{2}\right), y\left(r_{2}\right)\right)-f_{1}\left(r_{1}, x\left(r_{1}\right), y\left(r_{1}\right)\right)\right\|+ \\
& \left.\quad+B_{\alpha} \int_{r_{1}}^{r_{2}} \| f_{1}(s, x(s), y(s))\right) \| d s+\sum_{r_{1} \leq t_{k} \leq r_{2}}\left(\left\|I_{k}(x, y)\right\| .\right.
\end{aligned}
$$

This implies that $\left\|N_{1}\left(x\left(r_{2}\right), y\left(r_{2}\right)\right)-N_{1}\left(x\left(r_{1}\right), y\left(r_{1}\right)\right)\right\| \rightarrow 0$ whenever $r_{2} \rightarrow r_{1}$. As a consequence of Steps 1 to 3 together with the Arzelà-Ascoli, we conclude that $N$ maps $B_{q}$ into a precompact set in $P C(J, \mathbb{R}) \times P C(J, \mathbb{R})$.

Similarly, we have

$$
\begin{aligned}
& \left\|N_{2}\left(x\left(r_{2}\right), y\left(r_{2}\right)\right)-N_{2}\left(x\left(r_{1}\right), y\left(r_{1}\right)\right)\right\| \leq \\
& \leq A_{\beta}\left\|f_{2}\left(r_{2}, x\left(r_{2}\right), y\left(r_{2}\right)\right)-f_{2}\left(r_{1}, x\left(r_{1}\right), y\left(r_{1}\right)\right)\right\|+ \\
& \left.+\quad B_{\beta} \int_{r_{1}}^{r_{2}} \| f_{2}(s, x(s), y(s))\right)\left\|d s+\sum_{r_{1} \leq t_{k} \leq r_{2}}\right\| \bar{I}_{k}(x, y) \| .
\end{aligned}
$$

Again, by utilizing the Arzelà-Ascoli theorem we observe that $N_{2}$ is completely continuous. Therefore, we get $\left\|N_{2}\left(x\left(r_{2}\right), y\left(r_{2}\right)\right)-N_{2}\left(x\left(r_{1}\right), y\left(r_{1}\right)\right)\right\| \rightarrow$ 0 whenever $r_{2}$ tends to $r_{2}$. Thus, $N$ is completely continuous operator.

Step 4. It remains to show that

$$
\begin{aligned}
& \mathcal{A}=\{(x(\cdot), y(\cdot)) \in P C(J, \mathbb{R}) \times P C(J, \mathbb{R}): \\
&(x(\cdot), y(\cdot))=\lambda N(x(\cdot), y(\cdot)), \lambda \in(0,1)\}
\end{aligned}
$$

is bounded.

Let $(x, y) \in \mathcal{A}$. Then $x=\lambda N_{1}(x, y)$ and $y=\lambda N_{2}(x, y)$ for some $0<\lambda<1$. Thus, for $t \in[0, b]$, we have 


$$
\begin{aligned}
|x(t)| \leq & \left|x_{0}\right|+A_{\alpha}\left|f_{1}(t, x(t), y(t))\right|+B_{\alpha} \int_{0}^{t}\left|f_{1}(s, x(s), y(s))\right| d s \\
& +\sum_{0 \leq t_{k} \leq t}\left|I_{k}\left(x\left(t_{k}\right), y\left(t_{k}\right)\right)\right| \\
\leq & \left\|x_{0}\right\|+A_{\alpha}\left[c_{1}\|x\|+c_{2}\|y\|\right]+B_{\alpha} T\left[c_{1}\|x\|+c_{2}\|y\|\right] \\
& +\sum_{k=1}^{m}\left[d_{1 k}\|x\|+d_{2 k}\|y\|\right]
\end{aligned}
$$

and

$$
\begin{aligned}
|y(t)| \leq & \left|y_{0}\right|+A_{\beta}\left|f_{2}(t, x(t), y(t))\right|+B_{\beta} \int_{0}^{t}\left|f_{2}(s, x(s), y(s))\right| d s \\
& +\sum_{0 \leq t_{k} \leq t}\left|\bar{I}_{k}\left(x\left(t_{k}\right), y\left(t_{k}\right)\right)\right| \\
\leq & \left\|y_{0}\right\|+A_{\beta}\left[c_{3}\|x\|+c_{4}\|y\|\right]+B_{\beta} T\left[c_{3}\|x\|+c_{4}\|y\|\right] \\
& +\sum_{k=1}^{m}\left[e_{1 k}\|x\|+e_{2 k}\|y\|\right] .
\end{aligned}
$$

Therefore

$$
\begin{aligned}
|x(t)|+|y(t)| \leq & C+\left(A_{\alpha} c_{1}+B_{\alpha} T c_{1}+\sum_{k=1}^{m} d_{1 k}+A_{\alpha} c_{3}\right. \\
& \left.+B_{\alpha} T c_{3}+\sum_{k=1}^{m} e_{1 k}\right)\|x\| \\
& +\left(A_{\beta} c_{2}+B_{\beta} T c_{2}+\sum_{k=1}^{m} d_{2 k}+A_{\beta} c_{4}\right. \\
& \left.+B_{\beta} T c_{4}+\sum_{k=1}^{m} e_{2 k}\right)\|y\| \\
\leq & C+\max \left(\gamma_{1}, \gamma_{2}\right)(\|x\|+\|y\|), \quad \text { for all } t \in[0, T],
\end{aligned}
$$

where

$$
C=\left\|x_{0}\right\|+\left\|y_{0}\right\|
$$

and

$$
\begin{aligned}
& \gamma_{1}=A_{\alpha} c_{1}+B_{\alpha} T c_{1}+\sum_{k=1}^{m} d_{1 k}+A_{\alpha} c_{3}+B_{\alpha} T c_{3}+\sum_{k=1}^{m} e_{1 k}, \\
& \gamma_{2}=A_{\beta} c_{2}+B_{\beta} T c_{2}+\sum_{k=1}^{m} d_{2 k}+A_{\beta} c_{4}+B_{\beta} T c_{4}+\sum_{k=1}^{m} e_{2 k} .
\end{aligned}
$$


Hence

$$
\|x\|+\|y\| \leq C+\max \left(\gamma_{1}, \gamma_{2}\right)(\|x\|+\|y\|) .
$$

This implies that

$$
\|x\|+\|y\| \leq \frac{C}{1-\max \left(\gamma_{1}, \gamma_{2}\right)}:=K .
$$

Consequently

$$
\|x\| \leq K \quad \text { and } \quad\|y\| \leq K .
$$

This shows that $\mathcal{A}$ is bounded. As a consequence of Theorem 2 we deduce that $N$ has a fixed point $(x(\cdot), y(\cdot))$ which is a solution to the problem (1).

Step 5. Compactness of the solution sets. We will show that the set

$$
S=\{(x, y) \in P C \times P C,(x, y) \text { is solution of }(1)\}
$$

is compact.

Let $\left\{\left(x_{n}, y_{n}\right)\right\}_{n \in \mathbb{N}}$ be a sequence in $S\left(x_{0}, y_{0}\right)$. For every $n \in \mathbb{N}$, we get

$$
\begin{aligned}
x_{n}(t)= & x_{0}+A_{\alpha} f_{1}\left(t, x_{n}, y_{n}\right)+B_{\alpha} \int_{0}^{t} f_{1}\left(s, x_{n}(s), y_{n}(s)\right) d s \\
& +\sum_{0 \leq t_{k} \leq t} I_{k}\left(x_{n}\left(t_{k}\right), y_{n}\left(t_{k}\right)\right),
\end{aligned}
$$

and

$$
\begin{aligned}
y_{n}(t)= & y_{0}+A_{\beta} f_{2}\left(t, x_{n}, y_{n}\right)+B_{\beta} \int_{0}^{t} f_{2}\left(s, x_{n}(s), y_{n}(s)\right) d s \\
& +\sum_{0 \leq t_{k} \leq t} \bar{I}_{k}\left(x_{n}\left(t_{k}\right), y_{n}\left(t_{k}\right)\right),
\end{aligned}
$$

and set $B=\left\{\left(x_{n}, y_{n}\right): n \in \mathbb{N}\right\} \subset P C(J, \mathbb{R}) \times P C(J, \mathbb{R})$. From earlier parts of the proof of this theorem, we see that $B$ is bounded and equicontinuous. Then, from the Ascoli-Arzelà theorem, we can conclude that $B$ is compact. Hence, $\left(x_{n}, y_{n}\right)_{n \in \mathbb{N}}$ has a subsequence $\left(x_{n_{k}}, y_{n_{k}}\right)_{n_{k} \in \mathbb{N}} \subseteq S$ that converges to some $(x(\cdot), y(\cdot)) \in P C(J, \mathbb{R}) \times P C(J, \mathbb{R})$. Let

$$
\begin{aligned}
z_{x}(t)= & x_{0}+A_{\alpha} f_{1}(t, x, y)+B_{\alpha} \int_{0}^{t} f_{1}(s, x(s), y(s)) d s \\
& +\sum_{0 \leq t_{k} \leq t} I_{k}\left(x\left(t_{k}\right), y\left(t_{k}\right)\right),
\end{aligned}
$$

and

$$
\begin{aligned}
z_{y}(t)= & y_{0}+A_{\beta} f_{2}(t, x, y)+B_{\beta} \int_{0}^{t} f_{2}(s, x(s), y(s)) d s \\
& +\sum_{0 \leq t_{k} \leq t} \bar{I}_{k}\left(x\left(t_{k}\right), y\left(t_{k}\right)\right) .
\end{aligned}
$$


Then

$$
\begin{aligned}
\left|x_{n_{k}}-z_{x}(t)\right| \leq & A_{\alpha}\left|f_{1}\left(t, x_{n_{k}}, y_{n_{k}}\right)-f_{1}(t, x, y)\right| \\
& +B_{\alpha} \int_{0}^{t}\left|f_{1}\left(s, x_{n_{k}}(s), y_{n_{k}}(s)\right)-f_{1}(s, x(s), y(s))\right| d s \\
& +\sum_{0 \leq t_{k} \leq t}\left|I_{k}\left(x_{n_{k}}\left(t_{k}\right), y_{n_{k}}\left(t_{k}\right)\right)-I_{k}\left(x\left(t_{k}\right), y\left(t_{k}\right)\right)\right|,
\end{aligned}
$$

and

$$
\begin{aligned}
\left|y_{n_{k}}-z_{y}(t)\right| \leq & A_{\alpha}\left|f_{1}\left(t, x_{n_{k}}, y_{n_{k}}\right)-f_{2}(t, x, y)\right| \\
& +B_{\alpha} \int_{0}^{t}\left|f_{2}\left(s, x_{n_{k}}(s), y_{n_{k}}(s)\right)-f_{1}(s, x(s), y(s))\right| d s \\
& +\sum_{0 \leq t_{k} \leq t}\left|\bar{I}_{k}\left(x_{n_{k}}\left(t_{k}\right), y_{n_{k}}\left(t_{k}\right)\right)-\bar{I}_{k}\left(x\left(t_{k}\right), y\left(t_{k}\right)\right)\right| .
\end{aligned}
$$

Since $f_{1}(., .,),. f_{2}(., .,),. I_{k}(\cdot)$ and $\bar{I}_{k}(\cdot)$ are continuous functions, then as $n_{k} \rightarrow \infty, x_{n_{k}} \rightarrow z_{x}(t)$ and $y_{n_{k}} \rightarrow z_{y}(t)$, so

$$
\begin{aligned}
x(t)= & x_{0}+A_{\alpha} f_{1}(t, x, y)+B_{\alpha} \int_{0}^{t} f_{1}(s, x(s), y(s)) d s \\
& +\sum_{0 \leq t_{k} \leq t} I_{k}\left(x\left(t_{k}\right), y\left(t_{k}\right)\right),
\end{aligned}
$$

and

$$
\begin{aligned}
y(t)= & y_{0}+A_{\beta} f_{2}(t, x, y)+B_{\beta} \int_{0}^{t} f_{2}(s, x(s), y(s)) d s \\
& +\sum_{0 \leq t_{k} \leq t} \bar{I}_{k}\left(x\left(t_{k}\right), y\left(t_{k}\right)\right) .
\end{aligned}
$$

Hence, $S$ is compact.

\section{An EXAMPLE}

In this section we present an example to illustrate the usefulness and applicability of our results. Consider the following differential equation system 


$$
\left\{\begin{aligned}
D^{\frac{1}{2}} x(t) & =\frac{\sin (x(t)+y(t))}{16(\ln (t+1)+1)}+1, \quad t \in[0,1], t \neq \frac{3}{4} \\
D^{\frac{1}{2}} y(t) & =\frac{\sin x(t)+\sin y(t)}{18 \ln \left(t \exp \left(t^{2}\right)+1\right)}, \quad t \in[0,1], t \neq \frac{3}{4} \\
\Delta x\left(\frac{3}{4}\right) & =\frac{1}{5} \sin \left(x\left(\frac{3}{4}\right)+y\left(\frac{3}{4}\right)\right), \\
\Delta y\left(\frac{3}{4}\right) & =\frac{1}{7}\left[\cos \left(x\left(\frac{3}{4}\right)\right)+\cos \left(y\left(\frac{3}{4}\right)\right)\right] \\
x(0) & =\sqrt{3} \\
y(0) & =\sqrt{2}
\end{aligned}\right.
$$

Here

$$
\begin{aligned}
f_{1}(t, x, y) & =\frac{\sin (x(t)+y(t))}{16(\ln (t+1)+1)}+1, \\
f_{2}(t, x, y) & =\frac{\sin x(t)+\sin y(t)}{18 \ln \left(t \exp \left(t^{2}\right)+1\right)} .
\end{aligned}
$$

Clearly, the map $t \mapsto f_{1}(t, x, y)$ is jointly continuous for all $x, y \in \mathbb{R}$. The same for the map $f_{2}$. Also the maps $x \mapsto f_{1}(t, x, y)$ and $y \mapsto f_{2}(t, x, y)$ are continuous for all $t \in J$. Firstly, we show that $f_{1}, f_{2}, I_{1}$ and $\widetilde{I}_{1}$ are Lipschitz functions. Indeed, let $x, y \in \mathbb{R}$, then

$$
\begin{aligned}
\left|f_{1}(t, x, y)-f_{1}(t, \widetilde{x}, \widetilde{y})\right| & =\left|\frac{\sin (x(t)+y(t))}{16(\ln (t+1)+1)}-\frac{\sin (\widetilde{x}(t)+\widetilde{y}(t))}{16(\ln (t+1)+1)}\right| \\
& \leq \frac{1}{16}|x-\widetilde{x}|+\frac{1}{16}|y-\widetilde{y}| .
\end{aligned}
$$

Then

$$
\left|f_{1}(t, x, y)-f_{1}(t, \widetilde{x}, \widetilde{y})\right| \leq \frac{1}{16}|x-\widetilde{x}|+\frac{1}{16}|y-\widetilde{y}| .
$$

Analogously for the function $f_{2}$, we get

$$
\begin{gathered}
\left|f_{2}(t, x, y)-f_{2}(t, \widetilde{x}, \widetilde{y})\right| \leq \frac{1}{18}|x-\widetilde{x}|+\frac{1}{18}|y-\widetilde{y}|, \\
\left|I_{1}\left(x\left(\frac{3}{4}\right), y\left(\frac{3}{4}\right)\right)-I_{1}\left(\widetilde{x}\left(\frac{3}{4}\right), \widetilde{y}\left(\frac{3}{4}\right)\right)\right| \leq \frac{1}{5}|x-\widetilde{x}|+\frac{1}{5}|y-\widetilde{y}|, \\
\left|\widetilde{I}_{1}\left(x\left(\frac{3}{4}\right), y\left(\frac{3}{4}\right)\right)-\widetilde{I}_{1}\left(\widetilde{x}\left(\frac{3}{4}\right), \widetilde{y}\left(\frac{3}{4}\right)\right)\right| \leq \frac{1}{7}|x-\widetilde{x}|+\frac{1}{7}|y-\widetilde{y}| .
\end{gathered}
$$

Therefore the matrix

$$
M=\left(\begin{array}{ll}
0.26 & 0.26 \\
0.20 & 0.20
\end{array}\right)
$$

converges to zero, since its eigenvalues are $\lambda_{1}=0,46<1, \lambda_{2}=0<1$. From Theorem 3, the problem (13) has a unique solution. 


\section{Conclusion}

In this paper, we used the Perov and Schaefer fixed point theorems type in generalized Banach space to achieve the necessary criteria for the existence and uniqueness of the solution of considered coupled system of CaputoFabrizio fractional impulsive differential equations. Similarly, under particular assumptions and conditions, we have established the compactness of the solution sets of the system.

\section{ACKNOWLEDGEMENTS}

The authors would like to thank the referee and the editor for their careful reading and useful corrections and suggestions, which improved the quality of this paper.

\section{REFERENCES}

[1] S. Abbas, M. Benchohra, S. Hamani, J. and Henderson, Upper and lower solutions method for Caputo-Hadamard fractional differential inclusions, Mathematica Moravica, 23 (1) (2019), 107-118.

[2] B. Ahmad, S. K. Ntouyas, Existence results for a coupled system of Caputo type sequential fractional differential equations with nonlocal integral boundary conditions, Applied Mathematics and Computation, 266 (2015), 615-622.

[3] K. Aissani and M. Benchohra, Impulsive fractional differential inclusions with statedependent delay, Mathematica Moravica, 23 (2) (2019), 97-113.

[4] S. Aljoudi, B. Ahmad, J. J. Nieto, A. Alsaedi, A coupled system of Hadamard type sequential fractional differential equations with coupled strip conditions, Chaos Solitons Fractals, 91 (2016), 39-46.

[5] A. Alsaedi, D. Baleanu, S. Etemad, and S. Rezapour, On Coupled Systems of TimeFractional Differential Problems by using a New Fractional Derivative, Journal of Function Spaces, 2016 (2016), Article ID: 4626940, 8 pages.

[6] A. Alsaedi, S. Aljoudi, B. Ahmad, Existence of solutions for Riemann-Liouville type coupled systems of fractional integro-differential equations and boundary conditions, Electronic Journal of Differential Equations, 2016 (211) (2016), 1-14.

[7] N. Al-Salti,E. Karimov, K. Sadarangani, On a Differential Equation with CaputoFabrizio Fractional Derivative of Order $1<\beta \leq 2$ and Application to Mass-SpringDamper System, Progress in Fractional Differentiation and Applications, 2 (4) (2016), 257-263.

[8] M. Al-Refai, T. Abdeljawad, Analysis of the fractional diffusion equations with fractional derivative of non-singular kernel, Advances in Difference Equations, 2017 (2017), Article ID: 315, 12 pages.

[9] A. Atangana and B. S. T. Alkahtani, Analysis of the Keller-Segel model with a fractional derivative without singular kernel, Entropy, 17 (6) (2015), 4439-4453.

[10] S. M. Aydogan, D.Baleanu, A. Mousalou and S. Rezapour, On approximate solutions for two higher-order Caputo-Fabrizio fractional integro-differential equations, Advances in Difference Equations, 2017 (2017), Article ID: 221, 11 pages. 
[11] R. L. Bagley, P. J. Torvik, A theoretical basis for the application of fractional calculus to viscoelasticity, Journal of Rheology, 27 (1983), 201-210.

[12] M. Benchohra, J. Henderson, S. K. Ntouyas, Impulsive Differential Equations and Inclusions, Hindawi Publishing Corporation, 2 (2006), New York.

[13] H. Berrezoug, J. Henderson and A. Ouahab, Existence and uniqueness of solutions for a system of impulsive differential equations on the half-line, Journal of Nonlinear Functional Analysis, 2017 (2017), Article ID: 38, 16 pages.

[14] S. Bushnaq, S. Ali Khan, K. Shah and G. Zaman, Mathematical analysis of HIV/AIDS infection model with Caputo-Fabrizio fractional derivative, Cogent Mathematics and Statistics, 5 (2018), Article ID: 1432521, 12 pages.

[15] M. Caputo and M. Fabrizio, A new definition of fractional derivative without singular kernel, Progress in Fractional Differentiation and Applications, 1 (2) (2015), 73-85.

[16] D. Chalishajar, A. Kumar, Existence, Uniqueness and Ulam's Stability of Solutions for a Coupled System of Fractional Differential Equations with Integral Boundary Conditions, Mathematics, 6 (6) (2018), Article ID: 96, 12 pages.

[17] R. Darzi and B. Agheli, An analytical approach for systems of fractional differential equations by means of the innovative homotopy perturbation method, Mathematica Moravica, 22 (1) (2018), 93-105.

[18] A. D. Filip and A. Petruşel, Fixed Point Theorems on Spaces Endowed with VectorValued Metrics, Fixed Point Theory and Applications, 2010 (2010), Article ID: 281381, 15 pages.

[19] J. R. Graef, J. Henderson and A. Ouahab, Topological Methods for Differential Equations and Inclusions, CRC Press, Boca Raton, 2019.

[20] L. Hu, S. Zhang, Existence results for a coupled system of fractional differential equations with p-Laplacian operator and infinite-point boundary conditions, Boundary Value Problems, 2017 (2017), Article ID: 88, 16 pages.

[21] V. Lakshmikanthan, D. D. Bainov, P. S. Simeonov, Theory of impulsive differential equations, Singapore: World Scientific, 1989.

[22] J. Losada and J. J. Nieto, Properties of a new fractional derivative without singular kernel, Progress in Fractional Differentiation and Applications, 1 (2) (2015), 87-92.

[23] U. Riaz, A. Zada, Z. Ali, M. Ahmad, J. Xu, and Z. Fu, Analysis of nonlinear coupled systems of impulsive fractional differential equations with Hadamard derivatives, Mathematical Problems in Engineering, 2019 (2019), Article ID: 5093572, 20 pages.

[24] J. A. T. M. J. Sabatier, O. P. Agrawal, and J. T. Machado, Advances in Fractional Calculus, Theoretical Developments and Applications in Physics and Engineering, Springer, Dordrecht, The Netherlands, 2007.

[25] M. Seghier, A. Ouahab, and J. Henderson, Random solutions to a system of fractional differential equations via the Hadamard fractional derivative, The European Physical Journal Special Topics, 226 (16-18) (2017), 3525-3549.

[26] M. L. Sinacer, J. J. Nieto, A. Ouahab, Random fixed point theorem in generalized Banach space and applications, Random Operators and Stochastic Equations, 24 (2) (2016), 93-112. 
[27] A. Slama, and A. Boudaoui, Existence of solutions for nonlinear fractional impulsive stochastic differential equations with nonlocal conditions and infinite delay, International Journal of Differential Equations and Applications, 13 (4) (2014).

[28] A. Slama, and A. Boudaoui, Approximate controllability of fractional nonlinear neutral stochastic differential inclusion with nonlocal conditions and infinite delay, Arabian Journal of Mathematics, 6 (1) (2017), 31-54.

LABORATORY OF MATHEMATiCS

Modeling and Applications

UNIVERSITY OF ADRAR

ADRAR

Algeria

E-mail address: ahmedboudaoui@gmail.com

LABORATORY OF MATHEMATics

Modeling and Applications

UNIVERSITY OF ADRAR

ADRAR

Algeria

E-mail address: slama_dj@yahoo.fr 